\title{
A New Approach to Keyboard Inputting Error Prevention and Increasing Inputting Productivity
}

\author{
Lewis D. Eigen \\ Bea Enterprises Inc. \\ August 17, 2018
}

\begin{abstract}
This article describes the initial concept and developments of a new approach to reducing the number of inputting errors that are made in working with computers and decreasing the time the inputter must spend correcting errors. The approach involves intercepting and correcting errors before they are designated as such by Spell Check and eliminating the need for the time and effort of Spell Check. The operating principles and concepts of this approach, called Super ErrorCorrect ${ }^{\mathrm{TM}}$, is described, along with a software suite, that enables the implementation and the testing of the approach. This paper reports on the changes and evolution that resulted from analysis and limited Beta Testing. The preliminary data shows that not only is the Super ErrorCorrect ${ }^{\mathrm{TM}}$ approach feasible, but substantial time is saved while error rates are reduced markedly. Some data also suggests that in addition to the time saved in not using Spell Check, there is a tendency for users to type faster as they do not get negative reinforcement when they type faster and make errors as the software fixes the error in real time. Researchers are invited to collaborate in further research and licenses to the technology and software are provided at no cost if research results will be publicly disclosed.
\end{abstract}

Keywords: $\{$ typing, typographic errors, error prevention, keyboards, mistakes, error correction, typing speed, inputting, computers, spelling, ambiguous terms, English Language, input productivity, document production, writing\}

Author Note: Partial funding for this project is provided by the EA Foundation of Potomac, MD 


\section{Our New Approach}

We have developed a quasi-prevention approach which is independent of the user's state of spelling or touch-typing competence and does not depend in the user engaging in any special activities. Essentially, we have developed preliminary versions of software to intercept the errors, and the software corrects the errors "on the fly" - before they are designated by Spell Check or other equivalent software. Our draft software lends itself to manipulating a number of critical variables, not the least of which is what we call the "Correction Set". Technically we define a Correction Set to be a set of ordered pairs of strings $(A, B)$ where $A$ is not an English word, but $B$ is an English ${ }^{1}$ word. The software causes any instance of an $A$ to be replaced by the corresponding $B$.

\section{What is an English Word}

For our experimental purposes we had to first define what criteria we would use to determine whether a string of characters is or is not an English word. Most researchers have used a dictionary to make this determination. The dictionary, however, as a definition has flaws, not the least of which is that there are many well established dictionaries of the English language. There is even a Scrabble Dictionary to resolve the word question for players of that game.

Until recently, there were few other practical choices, but thanks to the National Science Foundation and Mark Davies, there is an excellent tool now available for making this determination. The general concept emanates from the concept of a "corpus" of a language. A corpus is a collection of documents in a language whereby each of the words that are used in any of the documents of the corpus can be extracted to the set of all words that appeared anywhere in the documents of the corpus. This list of words, if the corpus is large enough, has the following advantages over a dictionary definition.

- All forms of a word will likely appear in the corpus whereas they do not always appear in many dictionaries.

- The corpus is more objective as to what words are being used than is the collective opinion of a dictionary editorial group. The corpus is a record of what is and has been used as words whereas some dictionary editors allow "proper" English to color their decisions.

- The corpus set of words is readily available in machine form as opposed to dictionaries which may be online but cannot be incorporated into new experimental software without doing a lot of negotiating and often paying a large fee. Also, the dictionary is rarely available in standard formats where the corpora are.

\footnotetext{
${ }^{1}$ The concepts herein are not limited by language. All languages that are capable of having computer-generated documents lend themselves to this approach. However, all our initial experiments have been with English and it is that language which we will deal with in this paper.
} 
- The dictionaries are copyrighted while the best corpora, either are available to all under a Creative Commons license for a minor fee, or are in the public domain.

There have been a few different corpora created over the last few years, but the best and most recent one we think is the Davies corpus supported by the NSF and housed at Brigham Young University. ${ }^{2}$ As of 2017, the Davies corpus consisted of documents that contained 14 billion words. From this the corpus word set is produced and each of the words in the corpus set database also has a field which counts the frequency with which the particular word appeared among all 14 billion words. This word frequency count has an important use over and above the great value of the list of words which we use as a definition.

\section{Super ErrorCorrect ${ }^{\mathrm{TM}}$}

We have created an experimental software package and data sets we call Super ErrorCorrect ${ }^{\mathrm{TM}}$. This is a basic tool for experimenting with different approaches to the error correcting by machine approach. Essentially, this is how the software works with a word processor or other writing program:

As the user types text, the software intercepts each string of text that is typed and determines if that word is present in the Correction Set. If the string of text is NOT one of the $A$ terms of the $(A, B)$ type corrections in the Correction Set, nothing is done and the string is presumed to be a correct word and so is displayed as usual in the document. However, if the string matches any one of the A terms, then that string is an error and is not displayed. Instead the B term (the correct word) is displayed. So, as an example, if the string "corection" is typed, presumably it is not a word and will match one of the A pairs. So, instead of "corection" being displayed, the B word, "correction" will be displayed instead. The error "corection" in the normal case without a Super ErrorCorrect ${ }^{\mathrm{TM}}$ type approach would display, typically with a wavy red underline, indicating that it is an error. Then the typist would typically right-click the underlined error term, and he/she would be shown alternative possible corrections by Spell Check. The user then picks one, and that gets displayed. With the Super ErrorCorrect ${ }^{\mathrm{TM}}$ approach, the error term is infrequently displayed ${ }^{3}$, and the typist is then spared the effort of right-clicking the error term, deciding on the right correction and then clicking that. This is a significant productivity increase.

While this is the principle, it is, like so many things, easier said than done. Here are some of the problems and issues we found in setting up and configuring Super ErrorCorrect ${ }^{\mathrm{TM}}$.

${ }^{2}$ Mark Davies, (2017). iWeb: The Intelligent Web-based Corpus, Brigham Young University, https://corpus.byu.edu/corpora.asp

${ }^{3}$ The only time an error term is displayed is if there is no entry of that error term as an A term of the full correction set. 


\section{Problems and Issues with the Super ErrorCorrect ${ }^{\mathrm{TM}}$ Approach}

\section{The Size of the Correction Set}

If there is a small Correction Set used, many of the error terms that are typed will NOT be found in the A terms of the small Correction Set. In that case, the typed error will display and have the wavy red underline indicating that it is an error. In that case, the user must do what he/she does now without Super ErrorCorrect ${ }^{\mathrm{TM}}$-- use Spell Check. The important point is that even if the Correction Set is small, nothing is impaired if many incorrect terms are not fixed. The typist has received no gain from Super ErrorCorrect ${ }^{\mathrm{TM}}$, but has paid no penalty over the status quo ante-not using Super ErrorCorrect ${ }^{\mathrm{TM}}$.

The user is always better served by using the Super ErrorCorrect ${ }^{T M}$ method even if the Correction Set is small and few errors are prevented.

\section{The Concept of Orders of Corrections}

A typical input error is typing "formel" when "formal" was intended. Another error that is sometimes made when "formal" is intended is "fotmal" which is produced by, instead of typing the " $r$ " key, typing the adjacent " $\mathrm{t}$ " key. Or the inputter might have mistyped the " $\mathrm{f}$ " as a "g" and produced "gormal" instead of "formal". For every correct word, there are a number of possible errors that could be made by adding, deleting or changing some characters. Each of the three examples shows a different kind of error that the typist might make. However, the typist could also make two or more of these mistakes at the same time. "Gormel" illustrates two different errors-mistyping the " $g$ " for the " $f$ " AND the " $e$ " for " $a$ " vowel substation. We call this type of error a Second Order Error. This is in contrast to "gormal" where there was only one error made. These we call First Order Errors. And the term "gotmel" is a Third Order Error as all three errors are made in the same term.

In considering methods for analyzing or fixing input errors, the Error Order concept becomes very important for both practical and theoretical reasons. Consider a word $\Psi$ that has 5 possible First Order errors. It will also have 10 possible Second Order Errors, another 10 Third Order Errors, 5 Fourth Order Errors and 1 Fifth Order Error. This produces 31 different possible errors of all orders.

If a word had 10 possible First Order Errors (many long words would) then there are 1,023 possible errors of all orders. In the general case, if a word has $n$ First Order Errors possible then the total number of Errors of all orders is given by: $2^{n}-1$. A number that gets very large fast as $n$ increases.

A fundamental question is: If we are designing a system to correct errors, for which of the possible Error Orders should we try and correct? And what priority should we place on each order if we are going to correct the errors of that order?

If we were to cover all orders, we would be overwhelmed relatively early in the process. To guide us, we found very useful to do a "thought experiment": Consider an inputter of a word $\Psi$ with errors of $n$ degrees. The probability of the typist making an $\mathrm{n}^{\text {th }}$ order error is far lower than the likelihood of making 
a First or Second Order error. For example, if $n=6$, then to make a $6^{\text {th }}$ Order Error, the inputter would have to make ALL 6 possible errors in the same word at one timehighly unlikely. To make a $3^{\text {rd }}$ Order error, the person must make 3 different errors in the same word-much less likely than making only 1 or 2 but much more likely than

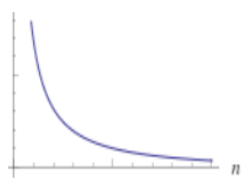
making 3. And making 4 would be less probable than making 5 and so on. The plot on the right illustrates the probability of error decreasing as the Error $\operatorname{Order}(n)$ increases. At the moment, we do not know the exact probabilities or even reasonable estimates of them, but the shape of the curve is clear.

The consequence of this little thought experiment, even without having good estimates or the probabilities is that it is much more important to have more different words corrected for the low order errors than it is to have higher order errors corrected for fewer words.

Our operational conclusion for the first stages of Super ErrorCorrect ${ }^{\mathrm{TM}}$ design was to concentrate only on First Order Corrections, and only to include any Second or higher order corrections when users tended to make those particular errors - when user demand / performance mandates it. The result is that all our work heretofore has only included First Order corrections with the occasional exception of a few higher order corrections suggested / demanded by Beta users.

\section{Input errors are not random.}

Some types of errors are made more frequently than others. For example, a very common typographic error is to juxtapose " $s$ " with an "a". They are adjacent keys on the standard keyboard. "a" and " $p$ " can be juxtaposed also, but that is very rare. They are far apart and typed with different hands.

Unfortunately, there is not very much research about the frequency of different typographic errors. Some like the "a" and "s" juxtaposition are so common that it is recognized by most people studying input error correction. This lack of research makes it difficult to prioritize the different error terms that we should enter into our Correction Set.

The main reason that there has been little such research, I believe, is that in past years, there was little one could do with such information if one had it. Typing has been around for well over a century but it has only been recently that such information could be utilized for something practical.

So, we tend to make the same or similar mistakes when inputting. There is another factor which really tends to make the same correction terms useful to most of us. That is the vocabulary that we use. In contrast to typing errors, the vocabulary that English speakers and writers use has been very extensively studied. There are over 170,000 different words in the English language according to the Oxford English Dictionary. However, surprising to most of us, only 3,000 words are used in 95 percent of everyday writing-- common texts and speech like newspapers, blogs, most books, movies, etc. ${ }^{4}$ Even more counter-intuitive is that fact that the first 1,000 words are used in $89 \%$ of everyday writing (Ibid)

${ }^{4}$ Edward B. Fry, Jacqueline E. Kress, (2006). The Reading Teacher's Book Of Lists, 5th Edition (New York: Wiley/Jossey-Bass). 
The implication is that if we just had corrections for 1,000 words, we could eliminate 89 percent of the errors that typists will make. Fry and Kress provide the lists, so it would seem as though the Super ErrorCorrect ${ }^{\mathrm{TM}}$ method with only 1,000 terms corrected would accomplish most of our objectives. Alas, this is not so. The situation is subtler. The reason is that, we can eliminate $89 \%$ of the inputting errors if we could eliminate the errors made typing 1,000 different words. But to eliminate the errors for a particular word we usually need many more than one correction pair. For example, the table on the right shows 16 different errors (BadWords) that can be made when people try and type "about" (the GoodWord).

So, if hypothetically there were 16 possible errors for each of the 1000 words, we would need a Correction Set of 16,000 correction pairs. To cover the 3,000 most frequently used words, we might need 48,000 in our Correction Set.

\section{The Correction Set Size Barrier}

To make an individual correction, the computer must consider the typed string and compare it to all 48,000 of the A terms in the Correction Set to cover the 3,000 word list. Even though computers are very fast, each comparison takes a few operations and for each word typed, if we used the 16 possible errors per word, the computer must make

16 Errors for Typing "about"

bbout aboit aboiut abotu abouit acout adout agout anout aobut aoub aout asbout avout baout sbout several hundred operations. This would appear to slow down the word processor, leaving us with a tradeoff of performance speed verses average percentage of errors corrected.

However as with many things in digital technology, intuition is not to be trusted. In many of these computer functions where for each item in a set a number of operations must take place, the time it takes to perform the function for all elements of the set seems to be proportional to the number of elements in the set. So, if we double the number of corrections in the Correction Set, the time that it takes would seem to double. The computer mathematicians write this as: $\mathrm{O}(\mathrm{n})$. But mathematicians have come up with some very sophisticated algorithms - mathematical procedures whereby instead of the time being proportional to the number of corrections, it is proportional to the logarithm of the number of corrections. $\mathrm{O}(\log n)$ to the mathematicians. If this kind of algorithm is used we can increase the number of correction terms by a factor of 10 and only increase the time by a factor of 2 . We could increase the Correction Set size by a factor of 1000 and only increase the time by a factor of 4 . That means that 48,000 corrections would only take 4 times as long to process as 48 .

The de facto result is that if we can write the a computer program that looks through the Correction Set and makes the replacement if necessary with one of these $\mathrm{O}(\log n)$ algorithms, there is no practical limit to the size of the Correction Set.

\section{With an O(log n) algorithm, we could have millions of corrections in the Correction Set without slowing the world processor down noticeably.}




\section{Microsoft used $\mathrm{O}(\log \mathrm{n})$ algorithms}

Early in the development of Word and Office, Microsoft used a structure for its Autocorrect functionality that is an amazingly sophisticated version of what the software engineers call a Binary Search Tree-a mathematical structure that was invented in 1960. One of the many desirable characteristics of binary search trees is that all of the 3 major functions-adding corrections, deleting corrections, and searching for a correction-all operate at $\mathrm{O}(\log \mathrm{n})$ time. Although Microsoft has never used more than 1,000 elements for this Correction Set, this incredibly powerful capability has been built in since the beginning. ${ }^{5}$ As a result, we have conducted almost all our research with Microsoft Office. While there are other fine word processors and document generators, we have not been able to find others that have the $\mathrm{O}(\log \mathrm{n})$ capability that we know of. There may be others but much of the software is "protected" as many of the publisher considers their programs to be "trade secrets", and we just cannot learn enough to integrate our Super ErrorCorrect ${ }^{\mathrm{TM}}$ with their software.

Another facet of Microsoft Office is that most of the functionalities of Word have been generalized to many of the other Office programs. So, when we do our experiments with Microsoft Office, the Super ErrorCorrect ${ }^{\mathrm{TM}}$ process works not only with Word but for e-mail in Outlook, OneNote, PowerPoint, and even Excel.

Therefore, for those researchers who choose to use our Super ErrorCorrect ${ }^{\mathrm{TM}}$ technology for their research, we recommend that they do so with Microsoft Office unless they are very familiar with the other document production software.

\section{Creating Correction Sets}

Once it became clear that there was no practical limit to the size of the Correction Sets, our Correction Sets became larger and larger for different trials. The last Correction Set that we used had over 12,000 terms and we are currently building one of over 15,000 .

Initially, we perceived that our central problem was to balance the tradeoff between the number of correction pairs and the time that the software would take for basic operations. However, we soon realized that the central problem really was the generation of all the possible corrections for the words we wanted to cover - the 1,000 most common words, the 3,000 or other sets. Over the almost 5 years that we have been researching this area. We have utilized a number of different techniques for generating possible error terms. These are presented in the order that we created them and used them.

\section{A. STAFF EXPERIENCE GENERATION}

We wrote some small utility programs that we used with Microsoft Word whereby, if the user typed a term that produced a red, wavy, underline indicating an error, the user selected the error term and typed a key combination to run the utility program which saved the highlighted term as the A term of a correction pair, and opened a text box for the user to enter the correct word which was saved as the $B$ term. This was used in the course of our regular work and we slowly built up a set of correction pairs.

\footnotetext{
${ }^{5}$ Microsoft has since its inception tended to over develop its software to be able to handle the anticipated technology developments of the future even though there was little present value to the development. This seems to have been another case with this example
} 
Initially, I used the tool as I am a serious dyslexic and if nothing else, I felt I could improve my own personal productivity and that of other dyslectics who comprise 5-10 percent of the population. . $^{6,7}$ In a little over a year, I had created a little over 1000 correction pairs, and I found myself making only half the rate of errors that I had previously. We then gave the utility to two other staff members and in the next year we had just under 3,000 correction pairs. One important observation that we all discovered was that each of us tends to have our own pattern of errors. So, in the first days of using the tool we were adding new ones every hour or so. However, as we saved each correction, we never could make that error again so in a short time, the new error rate began to slow and this continued over time. The $6^{\text {th }}$ month of use produced far fewer new corrections than was produced in the first month.

\section{B. RESEARCH LITERATURE ON COMMON TYPING ERRORS}

We started to do a literature search for the few studies of common misspellings that had been done. These we then entered into the correction database and quickly added another 3,000 new corrections. Most of the literature tended to deal with many of the same spelling errors, and many of those we had already entered via I) Staff Experience Generation. Eliminating duplicates became a substantial problem and at each stage we developed more and more software to do this automatically. The classic problem was where two staff $X$ and $Y$ each developed their own Correction Sets, but when we wanted to merge both the Correction Sets of $\mathrm{X}$ and $\mathrm{Y}$ into our master Correction Set, there were duplicates usually in two of the three Correction Sets and not infrequently in all three. Then when we had each set of errors emanating from a professional literature argument, we had more deduplication that was needed. The deduplication software has kept getting more sophisticated as the duplication possibilities grew in complexity and size of sets of the ordered pair corrections.

\section{COMPUTER ALGORITHMS}

At this point in our project, we developed an insight that caused us to rethink our entire process of creating correction pairs. The realization that caused us to have this insight was that over time we would add several different correction pairs covering a number of different error terms for the same GoodWord. But the process was serendipitous. If a particular error for a GoodWord was in one list we used it; if there was another that resulted from one of our staff, that too was added. However, as we have seen on page 6 , a GoodWord could have many BadWords that would be typical misspelling. There might be 10 and we might have only had 4 or 7 .

We put some effort in trying to expand the sets of BadWord pairs for a particular GoodWord to include other possibilities. ${ }^{8}$ At first, two of us had developed an intuitive way of doing this but did not understand enough of what we were doing to specify and program the process. A formal method was needed.

\footnotetext{
${ }^{6}$ Roongpraiwan R1, Ruangdaraganon N, Visudhiphan P, Santikul K., (2002). Prevalence and clinical characteristics of dyslexia in primary school students. J Med Assoc Thai., Nov; 85 Suppl 4:S1097-103.

${ }^{7}$ Austin Learning Systems (2018), Dyslexia Facts and Statistics, http://www.austinlearningsolutions.com/blog/38dyslexia-facts-and-statistics.html

${ }^{8}$ In theory there are a huge number of possible BadWords for a good Word. The number is much larger than even the number of words that could be generated from the permutations of all the subsets of the letters of the good word. Then we could add the superset permutations.
} 


\section{Reversing the Paradigm}

When we review the literature on spelling and the teaching thereof. The emphasis is on starting the errors and teaching how to avoid making that mistake. The research literature is full of errors of different types and procedures for avoiding them. One major objective is to classify different kinds of errors and generate a rule to avoid the error. "I before E except after C or in Neighbor or Weigh" So the same rule may apply to preventing a particular error for many different GoodWords. We need the opposite however. We do not want to start with the BadWord and make them good, because we will not find all the significant BadWords for a particular GoodWord. So, we reverse the thinking. Our problem is to Start with the GoodWord and find all the Bad Words that are likely to be mistaken for the GoodWord. Just the opposite from what we and others have been doing heretofore.

The reason is that we have to create the set of correction pairs for the most common GoodWords. To accomplish this, we wanted to create a machine routine that would start with GoodWords and generate the most likely BadWords. We so far have used two different methods:
A. Rule based spelling error generators
B. Position-based typographic error generators

For A, we wrote programs to implement rules that were in the literature or that we created based on our experience. Here are 3 examples:

Rule 013: If the GoodWord ends in a vowel + "ys" create a BadWord replacing the last "s" with "ies"

Rule 19: If the GoodWord contains "ck" AND the following letter is an element of $\{e, i, y\}$, then generate a BadWord where the "ck" is replaced by "cc"

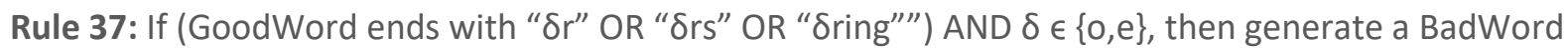
replacing the ending syllable " $\delta$ " with the OTHER element of $\{0, e\}$.

At this publication date, we had 35 different rules. If our software applies each of these rules in turn to the GoodWord, we may generate for each rule 1 or more BadWords, and for each of these BadWords, we can create a correction pair (GoodWord, BadWord) for the Correction Set. Because we run each rule sequentially, it is entirely possible for different rules to generate the same BadWord. This is yet another reason a duplication eliminator for the Correction Set is so critical.

We plan shortly on publishing the rules that we have by then created and asking for suggestions for additional or better rules.

A. generates correction pairs to cope with spelling errors. However, we use a different approach, B, to handle the typographic errors. The approach that we have taken is to have mathematical functions that transform each key on a standard keyboard to the set of most likely keys that are erroneously pressed instead of or in addition to the function argument. So, for any key $\delta, \psi(\delta)=$ the set of keys that are most likely to by inadvertently pressed. For convenience of operations we have used several functions to generate sets of alternative keys that are more or less frequently pressed in error. For example, one of the most common pure typographic errors is to press the key to the left or the right of the intended key. 
We call this function $\psi^{1}$. So, for example, $\psi^{1}(n)=\{b, m\}$ and $\psi^{1}(p)=\{0,[\}$. Note that we include the punctuation character keys and do not limit the set to alphabetical characters only. If someone types "[" and really meant to type the adjacent key " $p$ ", we want to correct it.

Not all typographic errors involve the 2 keys - the left and right of the intended key. Although it is with less probability, if one intends to type an " $n$ " he/she might, by accident, type " $h$ " or " $j$ ". These are the keys that are adjacent to the " $n$ " key but not on the same horizontal line. They are above on the next line of keys. So, we use the notation that, $\Psi^{2}(n)=\{n, j\}$. And $\psi^{2}(n)=\{1, ;, 9,-\}$. The " 2 " stands for the second degree of probability. Theoretically, we could extend this concept to additional degrees. However, just two orders produce a large number of potential corrections. Mathematicians denote the number of elements of a set $Z$ by $|Z|$. So, we see that $\left|\psi^{1}(\delta)\right| \leq 2$. Usually it is 2 but for example $\psi^{1}(a)=1$ because there is no key that might produce an error to the left of "a". However, for any $\delta,\left|\psi^{2}(\delta)\right| \leq 4$.

If we generated an error for each degree 1 and degree 2 keys resulting from these functions, we might average about 4.5 corrections for each character in a GoodWord. So, a 6 letter GoodWord would produce an average of 27 typographic correction pairs plus, say, 8 more from the spelling rules. This gives us a Correction Set of 35n pairs for $n$ GoodWords if the average world were 6 characters long. Applying this to our 3,000 Good Word base, we would end up with 105,000 correction pairs. This number is not out of the question and although it seems huge, it could be stored in a $3.6 \mathrm{~GB}$ file and not slow down Word or other app by any significant amount as long as the structure was a binary search tree or some $\mathrm{O}(\log \mathrm{n})$ equivalent. ${ }^{9}$

\section{BETA TEST USER EXPERIENCE}

When we started some Beta testing, some of the Beta testers communicated with us providing us with their view of the two classic kinds of errors:

I. Corrections that were not in the system but the user thought that they should have been. The result was that instead of Super ErrorCorrect ${ }^{\mathrm{TM}}$ correcting that particular error, it just displayed it with a wavy red underline and it had to be fixed with Spell Check

II. Corrections that were made where the user felt that they should not have been made. These mostly turned out to be our typographical errors in assembling the Correction Set. But there were others as well. Particularly noteworthy, were corrections where the BadWord we had used turned out to be a legitimate English word. In addition there were some corrections made where there were what I have termed "Ambiguous Terms" which we discuss next.

\footnotetext{
${ }^{9}$ These numbers are based on rough estimates we have made from the relatively little experience we have had. One of our objectives in sharing our data and techniques with other bonafide researchers is to obtain actual accurate estimates of these numbers. There are dozens of mini studies that need doing that would help advance the field-far too many for one small research group as ours.
} 
The Beta user feedback produced many of the Type I errors as defined above, and these caused us to add a correction pair to the Correction Set. This was a particularly rich source of additional corrections. The graph to the right shows the proportion of the next 1,000 corrections that were added after we started the Beta test with 20 people. These proportions are very variable at the experimental stage. If we come

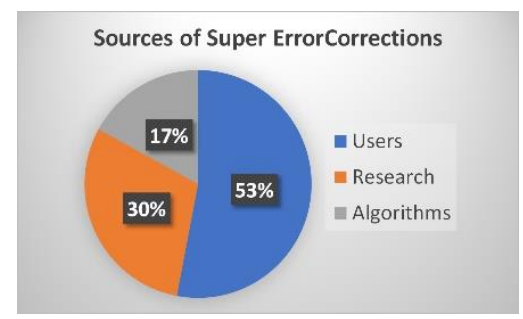
up with some good new algorithms, we will have a burst of corrections generated by them. However, the user feedback was so useful that we are building into the next version of Super ErrorCorrect ${ }^{\mathrm{TM}}$ an easy facility for the user to identify what he/she thinks is a Type I or Type II error, automatically send it to our server and we have a system to process the suggestions when they arrive, and if our editors approve, automatically add the Type I error to the Correction Set and delete the Type II errors therefrom.

\section{The Ambiguous Term Problem}

When we first started examining user suggestions for additional corrections, we began to observe a problem that we had not earlier considered. It is illustrated by the suggestion that we should include the correction pair (achive, achieve). "Achive" is a misspelled form of "Achieve" where the inputter forgot to include the " $\mathrm{e}$ " after the "I". We were about to add (achive, achieve) to our Correction Set, when one of our staff made the following observation: "Achive" is ALSO a simple misspelling of "Archive" where the typist forgot to add the " $r$ " after the " $A$ ". So, to what word do we correct "achive"? "archive" or "achieve". There seems to be no way of telling what the inputter intended. All other things being equal, whichever we choose, we have a 50-50 chance of being wrong. Another example is "onservation". Was it an error for "conservation" or "observation"? Another "Hobson's Choice".

We initially decided, based on one of the principles of the Hippocratic Oath, even today taken by many physicians: "Do no harm!" If we choose one of the two possible GoodWords, we would do harm about half the time. That was unacceptable. I choose "Ambiguous Terms" to describe this situation where we had a BadWord with two possible GoodWords to which we might correct them. Our decision therefore, was:

Do not attempt to correct Ambiguous Words!

When we reached this conclusion, we started to examine the implications of that decision. By not correcting Ambiguous Terms, the result would be that the uncorrected Ambiguous Term would be displayed as a spelling error with the wavy red underline. Then the user could use Spell Check to make the decision of which word he/she originally intended and make the correction. Essentially, our attempt to correct errors might work for a huge number of errors but not the Ambiguous Terms.

The question that we naturally asked was, "if we developed Super ErrorCorrect" ${ }^{\mathrm{TM}}$ to function with all errors except Ambiguous Terms, what percentage of the errors made would we never correct?" We soon realized that such a relatively simple concept as an Ambiguous Term and a similarly elementary question was much more difficult to answer than it appeared. First, despite our combing the literature, there was 
almost nothing on this concept, even searching for synonym terms. We have found no one who has ever even tried to estimate the percentage of errors that typists make that are Ambiguous Terms. We further could not find any compilation of Ambiguous Terms. If the ratio of Ambiguous Terms to all errors is very high, the prospects of our entire approach to error correcting would not be very good. In contrast, if very small, then there would not be that significant barrier to our objective. Ambiguous Terms. Therefore, became and is a central focus of our research with Super ErrorCorrect ${ }^{\mathrm{TM}}$.

First, we needed formal definitions if we were going to study this new subfield. By the time we realized that, we had also discovered that there were Ambiguous Terms that had 3 possible correct words, and 4, 5 and the current "Champion" is "baw" with 16 we shown in the list on the right. There are probably other Ambiguous Terms that even have more GoodWords.

So, the formal verbal definition of an Ambiguous Term is:

An alphabetical string (ordered set of alphabetical characters) which is not itself a word but with the transformation of one letter of the string to another letter or with the addition at any place in the string of any letter this will produce a word, and there must be at least 2 such transformations that produce words.

We use the definition of a word that requires that the string be a word indexed in the Corpus described on page 3 .

We are currently working on a mathematical definition and structure for Ambiguous Terms, so that we can create a system for correcting many Ambiguous Terms using modern mathematical and computer techniques. This will be separately published in a few months.

\begin{tabular}{|l|}
\hline GoudWords \\
fram \\
Ambiguous \\
Term \\
"baw" \\
bad \\
bag \\
ban \\
bar \\
bat \\
bawd \\
bawl \\
bay \\
bow \\
jaw \\
law \\
maw \\
paw \\
raw \\
saw \\
yaw \\
\hline
\end{tabular}

- Lists Ambiguous Terms

- Estimates the number of Ambiguous Terms there are.

- Estimates the proportion of all errors made by inputters that are Ambiguous Terms

We have, as a result of our Beta tests, observed the proportion of Ambiguous Terms. The sample is so small that we would not even describe it as an estimate. We observed between 2 percent and 7 percent in small samples.

If the true proportion is in that range, then Super ErrorCorrect ${ }^{\mathrm{TM}}$ with a large enough Correction Set would be able to correct between 93 and 98 percent of all errors made. That would certainly be a large improvement over the existing alternatives which rely totally on Spell Checked.

\section{A Serendipitous Benefit}

Our intention in this project was to improve the quality of documents by eliminating errors and increasing inputter productivity by eliminating the 2 clicks and 1 decision that the typist must make each time he/she uses the Spell Check. 
However, in the Beta tests, some users reported, and we observed, that some began to type at a faster speed. What seemed to be happening is that most of us can type faster than we normally do. But when we do speed up beyond our usual rate, we start to make more mistakes. So, each of us finds a comfortable typing rate which gives them a good balance between speed and error rate. ${ }^{10}$

However, when Super ErrorCorrect ${ }^{\mathrm{TM}}$ was being used in the Beta tests, there was little visible penalty to typing faster than the normal, comfortable level. Although the inputter made more errors, they were corrected so fast that the typist never even noticed, in most cases, that they were made at all. We had some anecdotal reports of typing speed increases of 50 percent. Self-reporting of this kind, is notoriously unreliable. Formal studies are needed to confirm this reported effect. Not only is the order of magnitude of the effect very important to measure, but it would be very desirable to learn the shape of the improvement curve over time of use of a correction system of the Super ErrorCorrect ${ }^{\mathrm{TM}}$ type.

\section{We Seek Collaborative Researchers}

The progress we have made with this new approach to Input Error Correction, while interesting, and already quite useful, is small relative to the possibilities that have emerged from the accomplishments and speculations thus far. We will be pleased to share the details of our work with bonafide researchers who would like to explore this field. Also, we will provide free licenses to use our software and data sets for other researchers who will publish their findings. If interested, feel free to contact the author at leigen@bea-enterprises.com or 13012997900 X102.

\footnotetext{
${ }^{10}$ One interesting possible explanation of this phenomenon is to consider it from a Skinnerian perspective of reinforcement. Although, not intended as such, the display of the wavy red underline produced by Spell Check is image-wise a negative reinforcer. This is strengthened by the fact that the wavy red underline functions as a discriminative stimulus for the user to slow down his/her train or thought and make additional clicks and a decision. This is a stronger negative reinforcer and since the physical behavior almost always follows the display, the wavy red underline strengthens as a secondary negative reinforcer. The typist via operant conditioning soon slows his/her typing speed to avoid so many negative reinforcers. If we introduce a Super ErrorCorrect ${ }^{\mathrm{TM}}$ type structure $^{\mathrm{t}}$ into the environment, when the inputter types faster, he does not get the negative reinforcers in most cases as the errors are fixed in real time so they are not even noticed. The absence of negative reinforcement even at typing speeds that would normally produce these negative stimuli, simply allows the typist to speed up without being stimulated by the negative reinforcer. Therefore, the inputter types faster.
} 